\title{
Fecal microbiota transplantation alleviated Alzheimer's disease-like pathogenesis in APP/PS1 transgenic mice
}

\author{
Jing Sun ${ }^{1}$, Jingxuan $\mathrm{Xu}^{2}$, Yi Ling ${ }^{1}$, Fangyan Wang ${ }^{3}$, Tianyu Gong ${ }^{4}$, Changwei Yang ${ }^{4}$, Shiqing Ye ${ }^{4}$, Keyue Ye ${ }^{4}$,
} Dianhui Wei ${ }^{4}$, Ziqing Song ${ }^{4}$, Danna Chen ${ }^{4}$ and Jiaming Liü ${ }^{1,4}$

\begin{abstract}
Alzheimer's disease (AD) is the most common dementia in the elderly. Treatment for AD is still a difficult task in clinic. $A D$ is associated with abnormal gut microbiota. However, little is known about the role of fecal microbiota transplantation (FMT) in AD. Here, we evaluated the efficacy of FMT for the treatment of AD. We used an APPswe/ PS1dE9 transgenic (Tg) mouse model. Cognitive deficits, brain deposits of amyloid- $\beta$ (A $\beta$ ) and phosphorylation of tau, synaptic plasticity as well as neuroinflammation were assessed. Gut microbiota and its metabolites short-chain fatty acids (SCFAs) were analyzed by $16 \mathrm{~S}$ rRNA sequencing and ${ }^{1} \mathrm{H}$ nuclear magnetic resonance (NMR). Our results showed that FMT treatment could improve cognitive deficits and reduce the brain deposition of amyloid- $\beta$ (A $\beta$ ) in APPswe/ PS1dE9 transgenic (Tg) mice. These improvements were accompanied by decreased phosphorylation of tau protein and the levels of $A \beta 40$ and $A \beta 42$. We observed an increases in synaptic plasticity in the Tg mice, showing that postsynaptic density protein 95 (PSD-95) and synapsin I expression were increased after FMT. We also observed the decrease of COX-2 and CD11b levels in Tg mice after FMT. We also found that FMT treatment reversed the changes of gut microbiota and SCFAs. Thus, FMT may be a potential therapeutic strategy for AD.
\end{abstract}

\section{Introduction}

Alzheimer's disease (AD) is the most common dementia in the elderly. The accumulation of amyloid- $\beta(A \beta)$ and downstream pathological events (such as tau hyperphosphorylation, neuroinflammation, and synaptic loss) eventually lead to a gradual decline in cognitive function $^{1,2}$. Recent evidence has demonstrated that $\mathrm{AD}$ is associated with abnormal gut microbiota ${ }^{3,4}$. The abundance of Helicobacteraceae and Desulfovibrionaceae at the family level and Odoribacter and Helicobacter at the

\footnotetext{
Correspondence: Jiaming Liu (wzjiaming_liu@163.com)

'Department of Neurology, The Second Affiliated Hospital and Yuying

Children's Hospital of Wenzhou Medical University, Wenzhou, Zhejiang 325027, China

${ }^{2}$ Department of General Surgery, The Second Affiliated Hospital and Yuying Children's Hospital of Wenzhou Medical University, Wenzhou, Zhejiang 325027, China

Full list of author information is available at the end of the article.

These authors contributed equally: Jing Sun, Jingxuan Xu
}

genus level significantly increased in APPswe/PS1dE9 transgenic ( $\mathrm{Tg}$ ) mice compared with wild-type (WT) mice $^{5}$. Gut microbes played vital roles in the pathogenesis of $\mathrm{AD}^{6,7}$. The probiotic consumption positively affected cognitive function in $\mathrm{AD}$ patients via regulation of gut microbiota ${ }^{8}$. The combination of Acidobacteria and Bifidobacterium significantly delayed $\mathrm{AD}$ progression in 3xTg-AD mice ${ }^{9}$. Our previous studies demonstrated that probiotics exert positive effects on depression ${ }^{10}$, vascular dementia ${ }^{11}$, diabetes combined with cerebral ischemia ${ }^{12}$ and traumatic brain injury ${ }^{13}$ by regulating the microbiota. Furthermore, specific bacteria from gut microbes produce metabolites, such as short-chain fatty acids (SCFAs), which can exert beneficial effects against neuropsychiatric disorders. Specifically, the SCFA butyrate can alleviate cognition deficits ${ }^{14}$ and enhance BDNF expression ${ }^{15}$. Our previous studies also showed that butyrate could exert a protective effect against Parkinson's disease ${ }^{16}$,

\section{(c) The Author(s) 2019}

(c) (i) Open Access This article is licensed under a Creative Commons Attribution 4.0 International License, which permits use, sharing, adaptation, distribution and reproduction cc) in any medium or format, as long as you give appropriate credit to the original author(s) and the source, provide a link to the Creative Commons license, and indicate if changes were made. The images or other third party material in this article are included in the article's Creative Commons license, unless indicated otherwise in a credit line to the material. If material is not included in the article's Creative Commons license and your intended use is not permitted by statutory regulation or exceeds the permitted use, you will need to obtain permission directly from the copyright holder. To view a copy of this license, visit http://creativecommons.org/licenses/by/4.0/. 
depression ${ }^{17}$, and traumatic brain injury ${ }^{18}$ in mice. The levels of SCFAs are influenced by the composition of the microbiota $^{19}$ and may change with the occurrence of gut microbial dysbiosis ${ }^{20}$. Considering that $\mathrm{AD}$ is associated with the abnormal gut microbiota, microbiota-targeted interventions, such as fecal microbiota transplantation (FMT), which is introduced gut microbiota into the host, might represent a potentially attractive therapeutic option against $\mathrm{AD}$.

FMT from a healthy donor to a patient or diseased animal is a conventional therapeutic approach for the reestablishment of a healthy gut microbial community and has been shown to have beneficial effects ${ }^{21}$. Recent evidence has demonstrated that FMT from healthy donors reduced alcohol-induced anxiety and depression in an animal model of chronic alcohol exposure ${ }^{22}$. FMT from resilient rats to antibiotic-treated pseudo-germ-free mice significantly improved pain and depression-like phenotypes $^{23}$. FMT reduced gut microbial dysbiosis and alleviated physical impairment in PD mice ${ }^{24}$. FMT have neuroprotective effects. However, the effect of FMT on $\mathrm{AD}$ by regulating gut microbiota remain fully unclear.

In this study, we therefore aimed to further elucidate the neuroprotective effects of FMT on AD. To address this, we performed a comprehensive exploration of cognitive impairment, $A \beta$ accumulation, synaptic dysfunction, neuroinflammation, as well as the changes of gut microbiota and its metabolites in APPswe/PS1dE9 transgenic mice.

\section{Materials and methods Animals}

Male APPswe/PSEN1dE9 transgenic (Tg) mice, aged 6 months, and nontransgenic wild-type (WT) mice were obtained from the Model Animal Research Center of Nanjing University (Nanjing, China). The mice were housed in a controlled environment at standard room temperature under a 12:12-h light/dark cycle and had access to standard food and water ad libitum. All experiments were approved and conducted in accordance with the guidelines of the Institutional Animal Care Committee of Wenzhou Medical University, China.

\section{Experimental design}

Tg mice were randomly divided into two groups $(n=8$ per group): (1) AD model group (Tg) and FMT treatment group (Tg + FMT). WT mice were used as the control group. The mice of $\mathrm{Tg}+\mathrm{FMT}$ group were intragastrically administered with $0.2 \mathrm{ml}$ fresh fecal solution of WT mice once daily for 4 weeks. For FMT experiments, $200 \mathrm{mg}$ of stool (from WT mouse pellets) was daily collected from WT mice and resuspended in $5 \mathrm{ml}$ of PBS mixed with sterile normal saline, and then passed through a $20 \mathrm{~mm}$ filter to remove large particulate. The filtrate was centrifuged at $3000 \times g$ for $15 \mathrm{~min}$ and dissolved in physiological saline with a concentration of $400 \mathrm{mg} / \mathrm{ml}$ for transplantation, as previously described ${ }^{25,26}$. Before FMT treatment, mice were intragastrically administered with triple antibiotics $(1.25 \mathrm{mg} / \mathrm{L}$ vancomycin, $2.5 \mathrm{mg} / \mathrm{L}$ ampicillin, and $2.5 \mathrm{mg} / \mathrm{L}$ metronidazole) in $0.2 \mathrm{ml} /$ mouse daily for 3 days to remove indigenous gut microbes, as previously described with a modification ${ }^{27,28}$. Mice of WT and $\mathrm{Tg}$ were treated same dose of physiological saline.

\section{Behavioral tests}

After the 4 weeks, Morris water maze (MWM) test and object recognition test (ORT) were performed to evaluate the cognitive function in mice.

\section{MWM test}

The MWM test was performed to assess spatial learning and memory ability using a cylindrical water tank (diameter $120 \mathrm{~cm}$, height $50 \mathrm{~cm}$, water depth $30 \mathrm{~cm}$ ) filled with water with a video capture system (DigBehv, Jiliang, China). The MWM was hypothetically divided into four quadrants. In the third quadrant, there was a diameter $6 \mathrm{~cm}$ platform hidden $1.0 \mathrm{~cm}$ under the water surface. During 5 consecutive days of continuous training, mouse was trained to locate the hidden platform from the starting point with the deadline of $60 \mathrm{~s}$. If the mouse did not find the platform within $60 \mathrm{~s}$, it was allowed to stay on the platform for $10 \mathrm{~s}$. On the 6th day, the platform was removed from the tank and the probe trial was performed. The mouse was allowed to swim freely for $60 \mathrm{~s}$ and the numbers of the platform crossing and the time spent in target quadrant was recorded.

\section{ORT}

ORT is based on the tendency of mice to discriminate a familiar from new object. The test was conducted 5 consecutive days and consisted of three phases: habituation, training and testing. Initially, mice were individually habituated to an open-field box $(25 \mathrm{~cm} \times 25 \mathrm{~cm} \times 35 \mathrm{~cm})$ for two consecutive days. During the training phases, on days 3 and 4, two objects of the same material were placed in a symmetric position in the center of the area. On day 5 , mice were returned to the area with one familiar object $(3 \mathrm{~cm} \times 2 \mathrm{~cm} \times 3 \mathrm{~cm}$ cuboid) and one novel object $(2 \mathrm{~cm}$ in diameter and $3 \mathrm{~cm}$ in height cylinder). The mouse was allowed to freely explore the object for $5 \mathrm{~min}$ at each time. All objects and apparatus were cleaned with $70 \%$ ethanol after each trial to eliminate residual odor. The time spent exploring the familiar object (TF) and the time spent exploring the novel object (TN) were recorded and analyzed. The exploration was defined as sniffing (within $1 \mathrm{~cm}$ ), pawing, or biting the object, but not leaning against or standing on the object. A discrimination index (DI) was calculated as follows: $\mathrm{DI}=(\mathrm{TN}-\mathrm{TF} / \mathrm{TN}+\mathrm{TF}) \times 100 \%$. 


\section{Tissue collection}

After the behavioral tests, mice were deeply anesthetized with $10 \%$ chloral hydrate $(5 \mu \mathrm{l} / \mathrm{g})$ and euthanized by cervical dislocation. We quickly dissected the brains and separated the cortex and hippocampus, then placed them in liquid nitrogen for rapid freezing and stored at $-80^{\circ} \mathrm{C}$ for western blotting. At the same time, tissue samples of the brain from mice in each group were collected and fixed, paraffin-embedded, and sectioned at $5 \mu \mathrm{m}$ for pathological staining. Fecal samples were obtained and stored at $-80^{\circ} \mathrm{C}$ for $16 \mathrm{~S}$ rRNA gene sequencing and nuclear magnetic resonance (NMR) spectroscopy.

\section{Analysis of $A \beta 40$ and $A \beta 42$ levels by ELISA}

The hippocampus samples of brain were homogenized and used to measure the concentration of $A \beta 40$ and $A \beta 42$ by using ELISA kit (Invitrogen Corporation, Camarillo, California, USA) according to the instructions. The standard curve was established and then used to calculate the levels of $A \beta 40$ or $A \beta 42$ in the tissues. The obtained values were corrected for the wet weight of brain sample and expressed as $\mu \mathrm{g} / \mathrm{mg}$.

\section{Western blotting}

The cortex samples of brain were homogenized using a handheld homogenizer in RIPA lysis buffer consisting of $50 \mathrm{mM}$ Tris (pH 7.4), $150 \mathrm{mM} \mathrm{NaCl}, 1 \%$ sodium deoxycholate, $0.1 \%$ SDS, and multiple inhibitors. The homogenate was incubated on ice for $20 \mathrm{~min}$ and centrifuged at $12,000 \times g$ for $20 \mathrm{~min}$ at $4{ }^{\circ} \mathrm{C}$. An enhanced bicinchoninic acid (BCA) protein assay kit was used to determine the protein concentration. The samples were separated using $12 \%$ sodium dodecyl sulfate-polyacrylamide gel electrophoresis (SDS-PAGE) and electro-transferred onto a PVDF membrane. The membrane was blocked in 5\% nonfat milk for $2 \mathrm{~h}$ at room temperature and then incubated with phospho-Tau231 (1:1000, Bioworld, Minnesota, USA), PSD-95 (1:1000, Bioworld, Minnesota, USA), and synapsin I (1:1000, Bioworld, Minnesota, USA) and COX2 (1:1000, Bioworld, Minnesota, USA) at $4{ }^{\circ} \mathrm{C}$ overnight. Subsequently, the membrane was washed in PBST and incubated with the appropriate HRP-conjugated secondary antibody (1:2000, Beyotime, China) for $1 \mathrm{~h}$ at room temperature. Images were scanned and the results were quantified. $\beta$-Actin and GAPDH was used as the loading control.

\section{Pathological staining}

Brain tissues were quickly dissected and fixed in $4 \%$ paraformaldehyde for $72 \mathrm{~h}$ after perfusion with $0.1 \mathrm{~mol} / \mathrm{L}$ phosphate buffer (containing 4\% paraformaldehyde), then dehydrated with ascending grades of ethyl alcohol, and cleaned in xylene and embedded in paraffin. The paraffinembedded tissues were sliced to a thickness of $5 \mu \mathrm{m}$ and dried at $65^{\circ} \mathrm{C}$. Slices were stained with Congo red staining solution (1:100, Solarbio, Beijing, China) for $10 \mathrm{~min}$, drip differentiation solution for about $20 \mathrm{~s}$, controlled by microscope, washed $5 \mathrm{~min}$; hematoxylin light dyeing for about $10 \mathrm{~s}$. The gradient alcohol was dehydrated, the xylene was transparent, and the neutral gum was sealed. Immunohistochemical staining was performed. PSD-95 (1:400, Bioworld, Minnesota, USA), synapsin I (1:200, Bioworld, Minnesota, USA), COX-2 (1:200, Bioworld, Minnesota, USA), and cluster of differentiation 11b (CD11b, 1:200, Southern Biotech, Birmingham, AL 35209, USA) were applied overnight at $4{ }^{\circ} \mathrm{C}$ and incubated with HRP-conjugated secondary antibodies and the sections were visualized using $\mathrm{DAB}$ as the chromogen.

\section{Fecal DNA extraction and 16S rRNA gene sequencing}

After all behavioral tests, animals were anesthetized. Fresh colonic fecal samples $(0.2-0.3 \mathrm{~g}$ per sample) were collected from the mice and immediately stored at $-80^{\circ} \mathrm{C}$. DNA extraction was performed using a QIAGEN stool DNA extraction kit (QIAGEN, Hilden, Germany) according to the manufacturer's instructions. For analysis of the phylogenetic composition of the gut microbiota, the V3-V4 region of the 16S rRNA gene was amplified using the Illumina MiSeq 2500 platform (Shanghai Majorbio Biopharm Technology Co., Ltd). A beta diversity distance matrix was computed from the previously constructed OTU table using UniFrac analysis. Principal component analysis (PCA), principal coordinate analysis ( $\mathrm{PCOA})$, a heatmap of RDA-identified key OTUs, and the unweighted pair group method with arithmetic mean (UPGMA) to analyze the beta diversity. The $16 \mathrm{~S}$ rRNA sequencing data set generated by the MiSeq platform was merged and decomposed into separate samples using QIIME version 1.9.0. OTUs were selected, and USEARCH V7 was used to search the Greengenes Database version 13.8, with a sequence similarity of $97 \%$. OTUs accounting for $<0.005 \%$ of the total number of sequences were excluded. The characteristics of the gut microbiota were analyzed by linear discriminant analysis (LDA) effect size (LEfSe) for biomarker discovery. LEfSe detects the features with significant differences in abundance using the Kruskal-Wallis rank sum test and applies LDA to evaluate the effect size of each feature.

\section{SCFA analysis by nuclear magnetic resonance (NMR) spectroscopy}

Fresh colonic fecal samples (50-60 mg per sample) were collected from mice and immediately extracted using $1 \mathrm{ml}$ phosphate buffer $(\mathrm{pH} 7.4)$ with $145.1 \mu \mathrm{M}$ TSP-d4 for chemical shift referencing $(\delta 0.00)$. The samples were frozen and thawed three times with liquid nitrogen, then homogenized (6500 rpm, 1 cycle, $60 \mathrm{~s}$ ) and centrifuged $\left(11,000 \times g, \quad 4{ }^{\circ} \mathrm{C}\right.$, and $\left.10 \mathrm{~min}\right)$. We transferred the 
supernatant to a new test tube and then added $600 \mu \mathrm{L}$ PBS to the pellets. The fecal supernatant was separated by centrifugation $\left(11,000 \times g, 4{ }^{\circ} \mathrm{C}\right.$, and $\left.10 \mathrm{~min}\right)$. A total of $600 \mu \mathrm{l}$ of spiked fecal extract was transferred to a $5 \mathrm{~mm}$ NMR tube (Norell, Morganton, NC). ${ }^{1} \mathrm{H}$ NMR spectra were obtained at $600.13 \mathrm{MHz}$ on a Bruker AVANCE III $600 \mathrm{MHz}$ NMR spectrometer with a 5-mm TXI probe (Bruker BioSpin, Rheinstetten, Germany). The ${ }^{1} \mathrm{H}$ NMR spectra were recorded by the standard single-pulse experiment with water signal presaturation. The main acquisition parameters were as follows: data points, $64 \mathrm{~K}$; relaxation delay, $2 \mathrm{~s}$; spectral width, $12,000 \mathrm{~Hz}$; and acquisition time, $2.65 \mathrm{~s}$ per scan, as previously described ${ }^{29}$.

\section{Statistical analysis}

Data are presented as the mean \pm SEM. Statistical analyses were performed using SPSS statistical software, version 18.0 (SPSS, Chicago, IL, USA). The data were analyzed by two-way ANOVA or one-way ANOVA by Tukey's test. $P<0.05$ was considered statistically significant.

\section{Results}

\section{Effect of FMT on cognitive deficits in Tg mice}

The cognitive function of mice was assessed by the MWM test and the ORT. The MWM test is conventionally used to measure cognitive function. In the training trials, all groups gradually learned to seek the hidden platform and gradually shortened their escape latency to reach the platform (Fig. 1a). The Tg mice exhibited prolonged escape latency compared with the WT mice (4th: $P<0.05$, 5th: $P<0.01$, Fig. 1a). Mice in the Tg + FMT group displayed shorter escape latencies than the Tg group on the 4th day and the 5th day (4th: $P<0.05$, 5th: $P<0.05$, Fig. 1a). Representative swimming paths of mice are illustrated in Fig. 1b. The Tg mice stayed in the target quadrant for a short time and crossed through it fewer frequency than the WT mice $(P<0.05$, Fig. 1c, d). The frequency and time spent in the target quadrant were much greater in the FMT treatment group than in the Tg group $(P<0.05$, Fig. $1 \mathrm{c}, \mathrm{d})$, indicating that FMT treatment was able to attenuate the impairment of spatial learning ability in Tg mice. In the ORT, the mice in the FMT treatment group also performed better than the Tg group $(P<0.05$, Fig. $1 \mathrm{e})$, as reflected by discrimination between the familiar and novel objects and a higher discrimination index in the FMT-treated mice.

\section{Effect of FMT on amyloid plaque and p-Tau level in $\mathrm{Tg}$ mice}

To investigate whether FMT affected $A \beta$ deposition in the Tg mice, we performed Congo red staining for compact amyloid plaques ${ }^{30}$. The number of brick-red patches distributed in the cortex and the hippocampus (CA1,
CA2/3, and DG) was counted in Congo red-stained brain tissue. The number of brick-red patches in the cortex and hippocampal regions (CA1, CA2/3, and DG) was higher in the Tg group than in the WT group (Fig. 1f). However, the number of brick-red patches was lower in the $\mathrm{Tg}+$ FMT group than in the Tg group (Fig. 1f), suggesting that FMT could result in a reduced amyloid plaque burden. $A \beta$ is the main component of amyloid plaques. The $A \beta 40$ level in the Tg group was significantly higher than the level in the WT group $(P<0.01$, Fig. 1g), whereas the level of A 440 in FMT-treated mice was dramatically decreased compared with the level in the Tg mice $(P<0.01$, Fig. $1 \mathrm{~g})$. Similarly, the level of A $\beta 42$ in FMT-treated mice was dramatically decreased compared with the level in the $\mathrm{Tg}$ mice $(P<0.01$, Fig. $1 \mathrm{~h})$. These results suggested that the amyloid burden could be altered in the Tg mice following treatment with FMT, based on the reduction in the A $\beta 42$ and $A \beta 40$ levels in the Tg mice. Western blots showed that Tau phosphorylation at the threonine 231 site was significantly decreased in the FMT-treated mice (Fig. 1i, j). These findings indicated that FMT could suppress $A \beta$ accumulation and Tau hyperphosphorylation in Tg mice.

\section{Effect of FMT on synaptic plasticity in Tg mice}

To examine whether FMT affected synaptic function in the $\mathrm{Tg}$ mice, we assessed synaptic plasticity-related proteins, including PSD-95 and synapsin I, by immunohistochemistry and Western blot. In immunohistochemistry, the level of PSD-95 in the cortex and hippocampal regions of the Tg group were lower than those in the WT group. However, the level of PSD-95 in the FMT group was higher than in the Tg group (Fig. 2a). Western blots further showed that the level of PSD-95 was significantly decreased in the Tg group compared with the WT group $(P<0.05$, Fig. 2 b, c) and significantly increased in FMTtreated mice $(P<0.05$, Fig. $2 \mathrm{~b}, \mathrm{c})$. In the Immunohistochemistry, the level of synapsin I in the cortex and hippocampal regions were lower in the Tg group than in the WT group. However, the level of synapsin I in the Tg + FMT group was higher than the level in the Tg group (Fig. 2d). Furthermore, western blots showed that the level of synapsin I in the Tg group were significantly lower than those in the WT group $(P<0.05$, Fig. $2 \mathrm{e}, \mathrm{f})$, whereas the FMT-treated mice had significantly increased level of synapsin I $(P<0.05$, Fig. 2 e, f). These findings indicated that FMT could attenuate synaptic dysfunction in $\mathrm{Tg}$ mice.

\section{Effect of FMT on COX-2 and CD11b in Tg mice}

COX-2 protein is critical in the pathophysiological process of inflammation. The level of COX-2 in the cortex and the hippocampal regions of the Tg group were higher than those of the WT group. However, the level of COX-2 in the $\mathrm{Tg}+\mathrm{FMT}$ group was lower than that in the $\mathrm{Tg}$ 
(A)

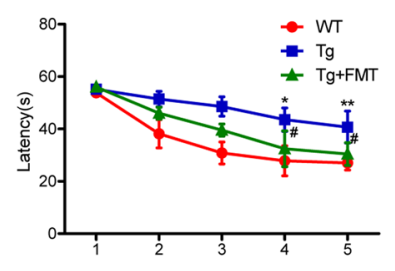

(C)

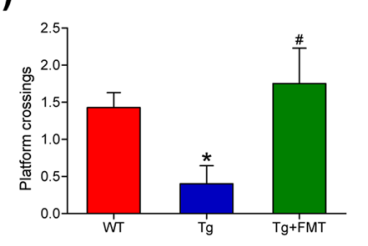

(B)

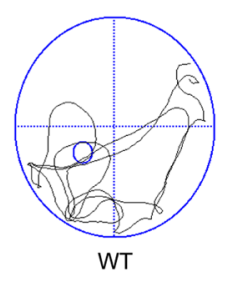

(D)

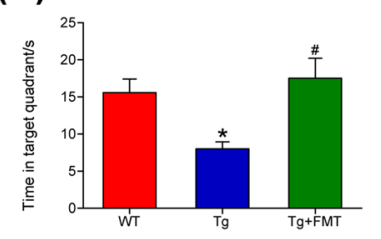

(E)

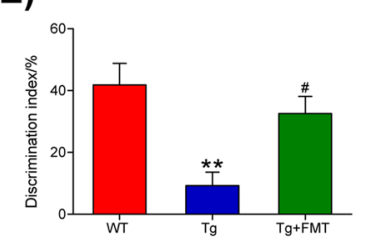

(F)
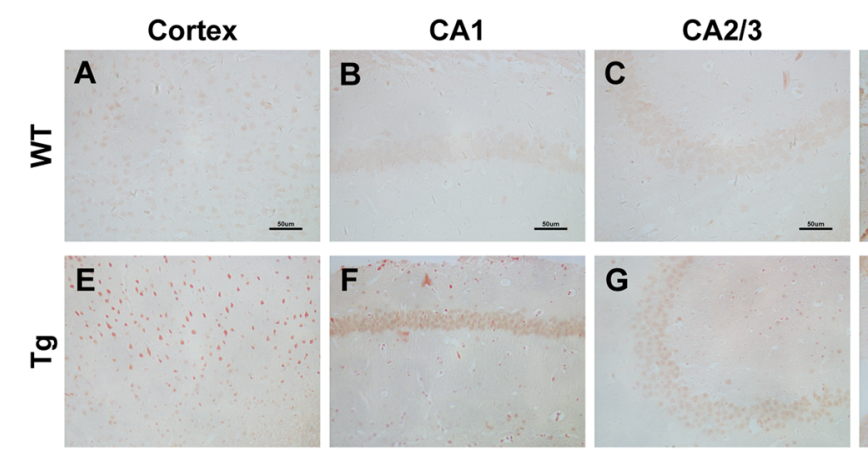

G
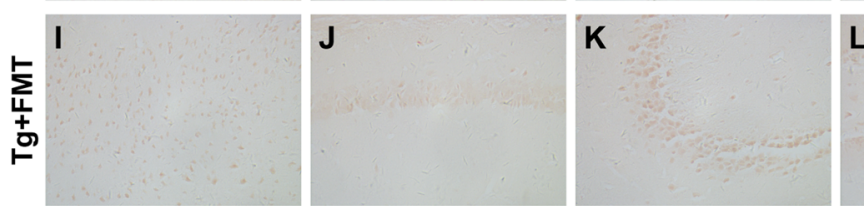

(G)

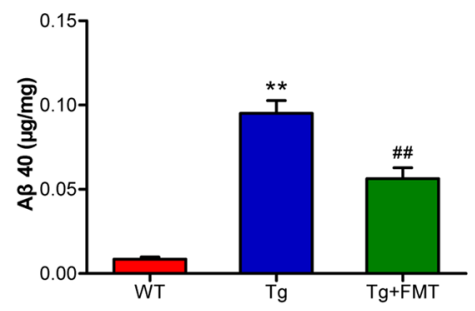

(H)

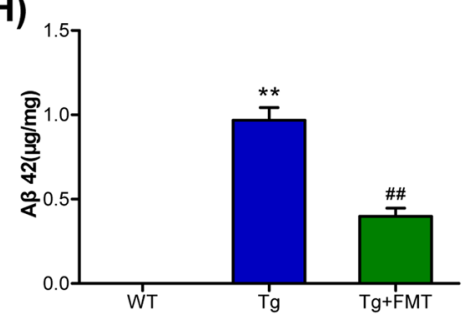

(I)

(J)
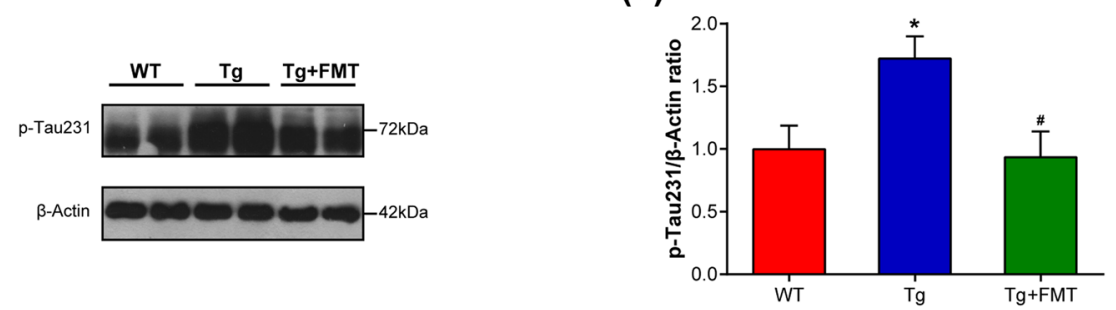
(see figure on previous page)

Fig. 1 Effects of FMT on behavioral function and amyloid plaque in Tg mice. a Escape latency of mice for 5 consecutive days in Morris water maze test. $\mathbf{b}$ Representative swimming tracks of mice in Morris water maze test. c Frequency of crossing the platform of mice in Morris water maze test. $\mathbf{d}$ Detention time in target quadrant in Morris water maze test. e Discrimination index of the object recognition test. Error bars indicate $S E M ; ~ n=$ 6-8 for each group. ${ }^{*} P<0.05$ vs. WT group, ${ }^{* *} P<0.01$ vs. WT group, ${ }^{\#} P<0.05$ vs. Tg group. $\mathbf{f}$ Representative photographs of Congo red staining in the cortex and hippocampal regions (CA1, CA3, and DG). CA, cornu ammonis; DG, dentate gyrus; magnification $\times 400$. Scale bar $=50 \mu \mathrm{m}$. $\mathbf{g}$ The level of $A \beta_{40}$ in the brain. $\mathbf{h}$ The level of $A \beta_{42}$ in the brain. $\mathbf{i}$ Western blot analysis of $p$-Tau expression. $\mathbf{j}$ Quantitative analysis ratio of $p$-Tau/ $\beta$-actin, the reference value of the $p$-Tau/ $\beta$-actin was the ratio of the WT group. Error bars indicate SEM; $n=4-6$ for each group. ${ }^{*} P<0.05$ vs. WT group, ${ }^{* *} P<0.01$ vs. WT group, ${ }^{\#} P<0.05$ vs. Tg group, ${ }^{\# \#} P<0.01$ vs. Tg group

group (Fig. 3a). Western blots further showed that the level of $\mathrm{COX}-2$ in the $\mathrm{Tg}$ group were significantly increased compared with the WT group $(P<0.05$, Fig. $3 b, c)$, whereas that of the FMT-treated mice were significantly decreased in the level of COX-2 $(P<0.05$, Fig. $3 \mathrm{~b}, \mathrm{c})$. The level of $\mathrm{CD} 11 \mathrm{~b}$ in the $\mathrm{Tg}$ group were higher than those in the WT group (Fig. 3d). However, the level of CD11b in the Tg + FMT group was lower than that in the Tg group (Fig. 3d). These findings indicated that FMT could attenuate neuroinflammation in Tg mice.

\section{Effect of FMT on the composition of the gut microbiota in Tg mice}

To investigate whether FMT affected the gut microbiota of the $\mathrm{Tg}$ mice, we performed microbiota analysis. Discriminant analyses using LEfSe showed that the treated and untreated mice had significantly different bacterial phylotypes (Fig. 4a). Analysis revealed differences in the abundance of taxa. Gut microbiota of $\mathrm{Tg}$ mice was characterized by an overabundance of Proteobacteria and Verrucomicrobia at the phylum level and Akkermansia, and Desulfovibrio at the genus level, as well as a low abundance of Bacteroidetes at the phylum level and the Bacteroidales_S24-7 group and Alloprevotella at the genus level, as displayed in Fig. 4b-f. At the phylum level, Bacteroidetes were enriched in the WT mice, while Proteobacteria and Verrucomicrobia were enriched in the Tg mice. The abundance of Proteobacteria and Verrucomicrobia was decreased, while that of Bacteroidetes was increased, in the FMT-treated mice (Fig. 4b). At the genus level, the abundance of the Bacteroidales_S24-7 group, Ruminococcaceae_UCG-01, and Alloprevotella were enriched in the WT mice, while Akkermansia and Desulfovibrio were enriched in the Tg mice. FMT reversed the alterations in the composition of the microbiota in $\mathrm{Tg}$ mice.

\section{NMR-based metabolic profile}

The NMR spectra were normalized into corresponding tissue weights to assess the relative content of the extracted and bound metabolites to reduce the dimensionality of the data for further metabolic analysis, as reflected in the typical ${ }^{1} \mathrm{H}$ NMR spectra of fecal samples and a total of three identified metabolites, the SCFAs acetate, propionate and butyrate, as shown in Fig. 5a. It was demonstrated from the PLS-DA score plot that the Tg group was separated from the other two groups along PLS1 (Fig. 5b). To examine whether FMT affected SCFAs levels in the Tg mice, we assessed the levels of acetate, propionate and butyrate. The level of butyrate was significantly increased in the FMT-treated mice (Fig. 5e), and the levels of acetate and propionate were not significantly different among the three groups (Fig. 5c, d).

\section{Discussion}

FMT has been shown to exert beneficial effects against neuropsychiatric disorders by modulating gut microbiota, however, its effect on AD remains unclear. This study demonstrated the neuroprotective effects of FMT against $\mathrm{AD}$ in APPswe/PS1dE9 transgenic mice. Our results showed that FMT treatment significantly relieved cognitive deficits, $A \beta$ accumulation, synaptic dysfunction, and neuroinflammation. These protective effects of FMT may be related to reversing alterations of gut microbiota and its metabolites SCFAs.

Cognitive deficits are the main manifestation of $\mathrm{AD}^{31,32}$. Improving cognitive function is a desirable target for therapies against $\mathrm{AD}$. Improving cognitive deficits in $\mathrm{AD}$ patients $^{33}$ and $\mathrm{Tg}$ mice ${ }^{9}$ via regulating gut microbiota is an area of active research. In this study, the data showed that FMT effectively alleviated cognitive deficits in the Tg mice as shown by the MWM test and the ORT, suggesting that FMT might be involved in alleviating cognitive deficits. The development of $\mathrm{AD}$ was accompanied by pathological changes. $A \beta$ deposition is a hallmark of $A D$ pathogenesis. Congo red staining is a reliable indicator of $\mathrm{A} \beta$ deposition, which is an important cause of cognitive decline in AD. Probiotics reduced the accumulation of $A \beta$ and ameliorated the effect of $A \beta$ toxicity ${ }^{34}$. In this study, the data showed that FMT treatment could decrease amyloid deposition and the levels of $A \beta 40$ and $A \beta 42$ in the Tg group. C-Jun $\mathrm{N}$-terminal kinase (JNK) is related to the production of $A \beta^{35,36}$. Inactivation of JNK could decrease $A \beta$ production and the resulting cognitive deficits in $\mathrm{AD}$ mice ${ }^{36,37}$. Our results showed that FMT treatment could inhibit JNK activity in Tg mice. Taken 
(A)

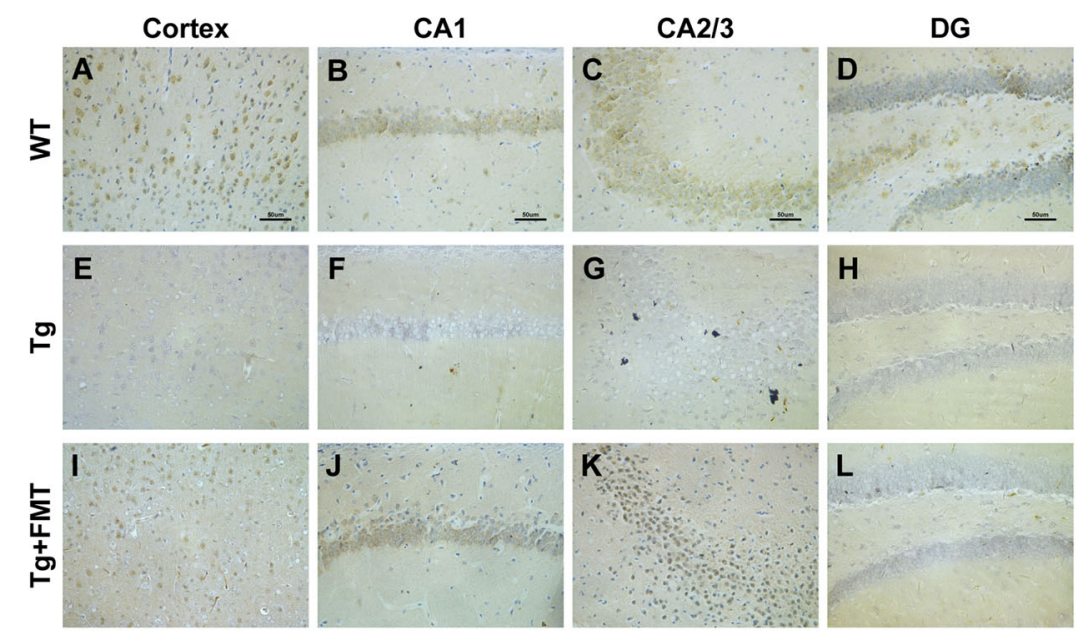

(B)

(C)
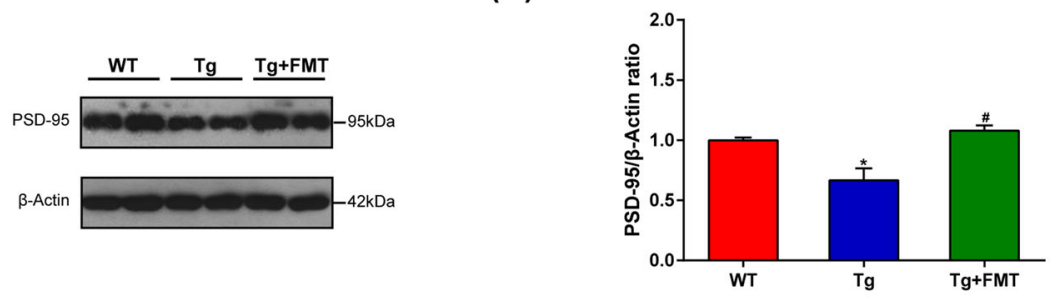

(D)
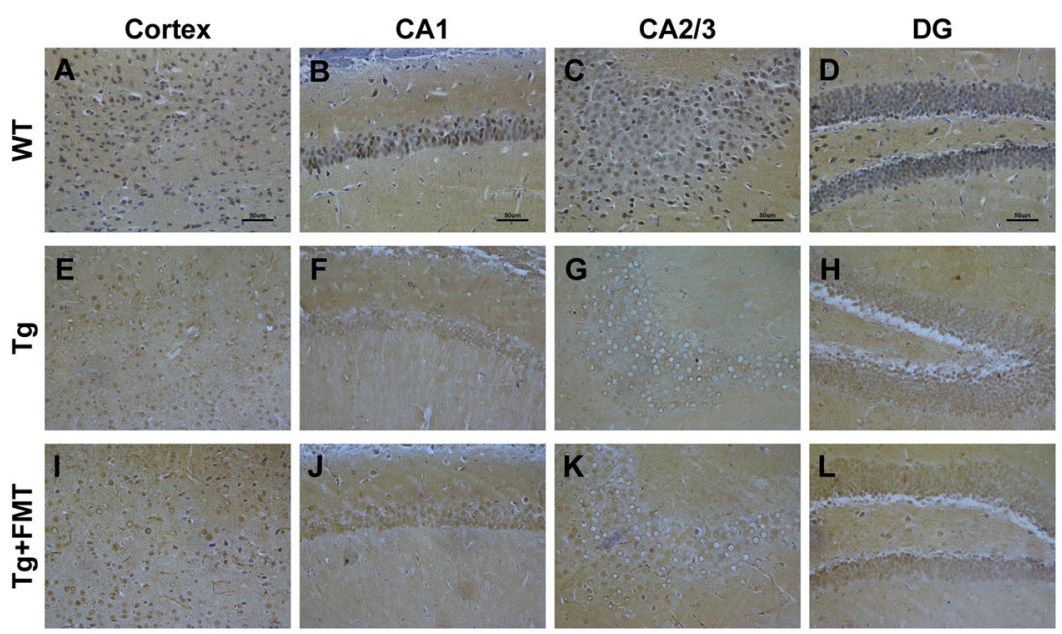

(E)

(F)
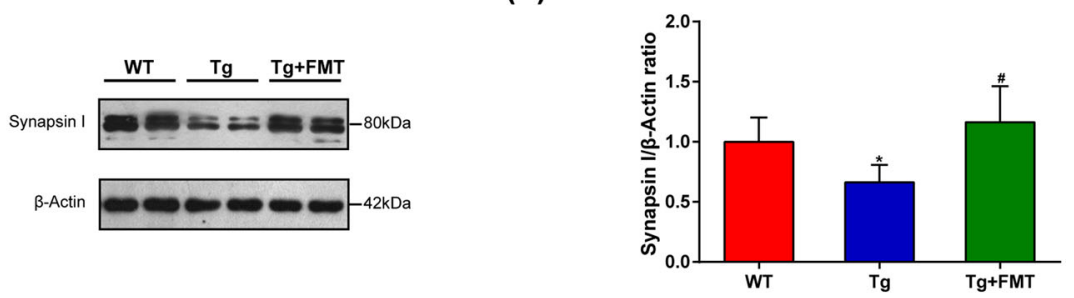

Fig. 2 (See legend on next page.) 
(see figure on previous page)

Fig. 2 Effects of FMT on levels of PSD-95 and synapsin I in Tg mice. a Immunohistochemical analysis of PSD-95 in the cortex and hippocampal regions (CA1, CA3, and DG); Magnification $\times 400$. Scale bar $=50 \mu \mathrm{m}$. b Western blot analysis of PSD-95 expression; (c) quantitative analysis of expression of PSD-95, the reference value of the PSD-95/ $\beta$-actin was the ratio of the WT group. $\mathbf{d}$ Immunohistochemical analysis of synapsin I in the cortex and hippocampal regions (CA1, CA3, and DG); Magnification $\times 400$. Scale bar $=50 \mu \mathrm{m}$. e Western blot analysis of synapsin I expression; (f) quantitative analysis of expression of synapsin I, the reference value of the synapsin I/ $\beta$-actin was the ratio of the WT group. Error bars indicate SEM; $n=4-6$ for each group. ${ }^{*} P<0.05$ vs. WT, ${ }^{\#} P<0.05$ vs. Tg

together, our results suggested that FMT treatment could ameliorate cognitive deficits, $A \beta$ deposition and Tau phosphorylation in $\mathrm{Tg}$ mice.

$\mathrm{AD}$ is mainly characterized by abnormal synaptic plasticity $^{7,38,39}$, which is strongly correlated with the cognitive decline observed in AD patients ${ }^{40}$. The accumulation of plaques could affect synaptic activity ${ }^{41,42}$, which was an early pathogenic event in AD. PSD-95 and synapsin I were indicators of synaptic plasticity ${ }^{43}$. PSD-95 was a common marker of postsynaptic components and indirectly reflects changes in synaptic plasticity ${ }^{44}$. Synapsin I was a presynaptic protein that responds to neuronal activity and regulates the availability of synaptic vesicles to participate in neurotransmitter release ${ }^{45}$. The levels of synapsin I and PSD-95 were significantly decreased in AD mice ${ }^{7,46}$. In this study, FMT treatment reversed the decreased levels of PSD-95 and synapsin I in the Tg mice, indicating that FMT treatment could prevent the abnormal synaptic plasticity of AD. Neuroinflammation was thought to be an important aspect of AD pathogenesis ${ }^{47}$. COX-2 activated proinflammatory signaling pathways ${ }^{48}$, and interactions between $A \beta$ and microglia induced the release of proinflammatory factors. FMT provided neuroprotection by inhibiting inflammation in an animal model of $\mathrm{PD}^{24}$. In this study, FMT treatment decreased the levels of COX-2 and $\mathrm{CD} 11 \mathrm{~b}$ in the Tg mice, indicating that FMT treatment could inhibit neuroinflammation in AD.

Alterations of gut microbiome were related to $\mathrm{AD}^{3}$. Gut microbiome could influence brain function via the gutbrain axis ${ }^{3,4,49,50}$. Recent evidence has demonstrated that gut microbiota of AD patients and animal models were altered $^{4,51,52}$. Germ-free (GF) mice showed significantly increased brain $A \beta$ levels after transplantation of fecal samples from $\mathrm{AD}$ mice ${ }^{3}$. Manipulating gut microbiota can influence $A \beta$ deposition and neuroplasticity processes $^{53,54}$. In this study, Proteobacteria, Verrucomicrobia, Akkermansia, and Desulfovibrio were enriched, and Bacteroidetes and Alloprevotella decreased in the Tg mice, whereas FMT reversed the alterations in the microbial composition. The abundance of Proteobacteria increases in the gut microbiome during aging ${ }^{55}$. AD pathogenesis in the Tg mouse model could shift the gut microbiota toward a higher abundance of Proteobacteria ${ }^{56}$. Moreover, Proteobacteria was related to inflammation, which was linked to $\mathrm{AD}$ pathogenesis ${ }^{57}$ and an increased risk of dementia ${ }^{58}$. In this study, FMT reversed the increase in Proteobacteria in the Tg mice. At the family level, the abundance of Desulfovibrionaceae was also elevated in $\mathrm{Tg}$ mice $^{56}$. In addition, the family Desulfovibrionaceae has a significantly negative correlation with spatial learning and memory ability, active avoidance response, and object recognition memory capability ${ }^{59}$. Unpredictable restraint stress potentiates rotenone-induced effects in the colon, including an increase in Akkermansia ${ }^{60}$. However, the changes associated $\mathrm{AD}$ are not consistent across $\mathrm{AD}$ studies. The phylum Verrucomicrobia, the genus Akkermansia genus and certain species are increased in PD patients $^{61,62}$. The APP/PS1 mouse model showed a distinct microbial signature, with a decreased abundance of Akkermansia $^{63}$. In this study, FMT reversed the decrease in Bacteroidetes in the Tg mice. Bacteroidetes has a wellknown and frequently discussed connection to $\mathrm{AD}$. $\mathrm{AD}$ patients showed a characteristic increase in Bacteroidetes $^{64}$. The abundance of Bacteroidetes was decreased in $\mathrm{AD}$ mice ${ }^{65}$. Another study found, in contrast to our results, that the abundance of Bacteroidetes increased in the microbiome of $\mathrm{AD}$ participants ${ }^{64}$. In this study, the overabundance of Bacteroidetes was restored in the FMTtreated Tg mice, and Bacteroidetes was able to trigger the generation of SCFAs ${ }^{66}$. In this study, the overabundance of Proteobacteria and Desulfovibrio and the low abundance of Bacteroidetes and Alloprevotella might have participated in the process of AD. Further studies are needed to identify functional metabolites.

SCFAs are among the vital chemical mediators of the gut-brain axis. SCFAs such as acetate, propionate and butyrate may exert neuroprotective effects, which are influenced by gut microbiome ${ }^{67}$. SCFAs may also modulate the maturation and function of microglia in the brain $^{68}$, suggesting the potential benefits of bacteriaderived SCFAs in modulating neuroinflammatory processes associated with neurodegenerative disorders. The SCFA butyrate could exert neuroprotective effects ${ }^{69}$. Butyrate and propionate also interfere with $A \beta_{1-40}$ oligomerization ${ }^{70}$. Bacterial generation of butyrate, as an inhibitor of histone deacetylase, was known to exert multiple effects against a variety of neuropsychiatric disorders $^{71}$. Our previous study also demonstrated that butyrate had neuroprotective effects on depression, PD and traumatic brain injury in mice ${ }^{16-18,72}$. In this study, 
(A)
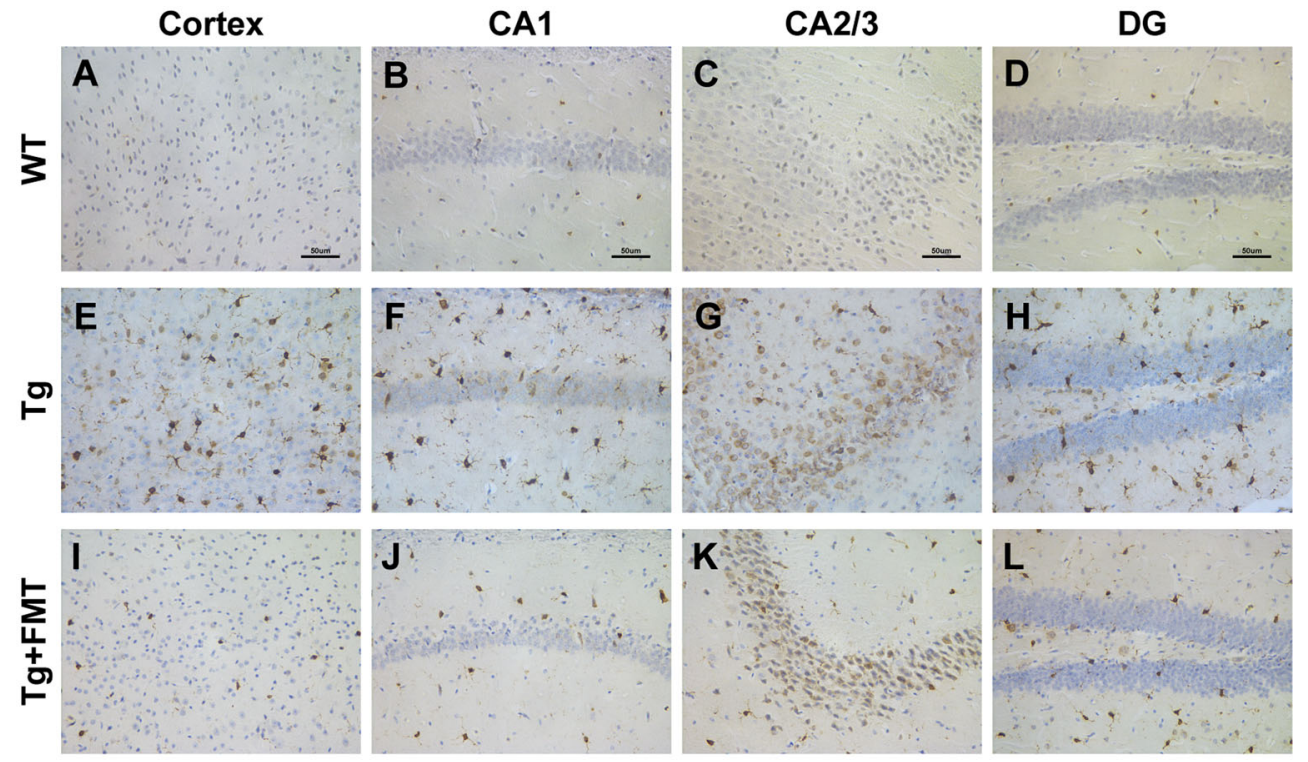

(B)

(C)
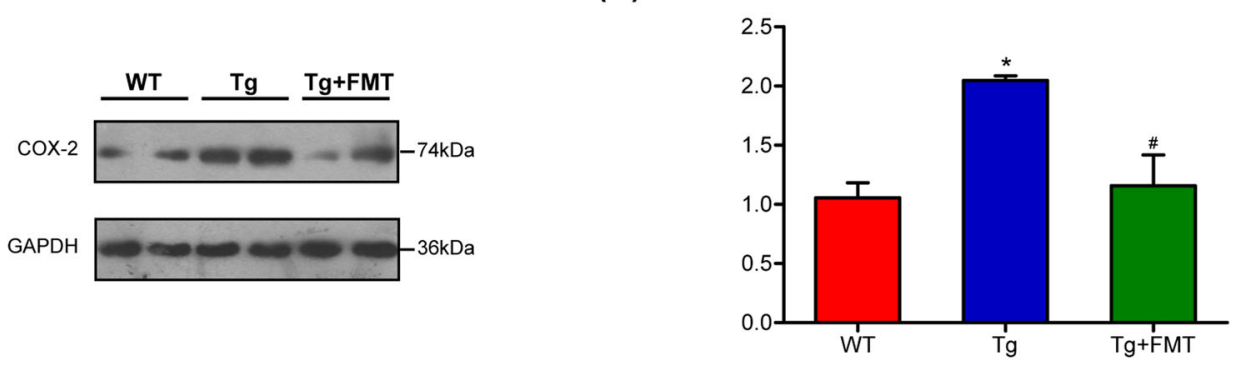

(D)
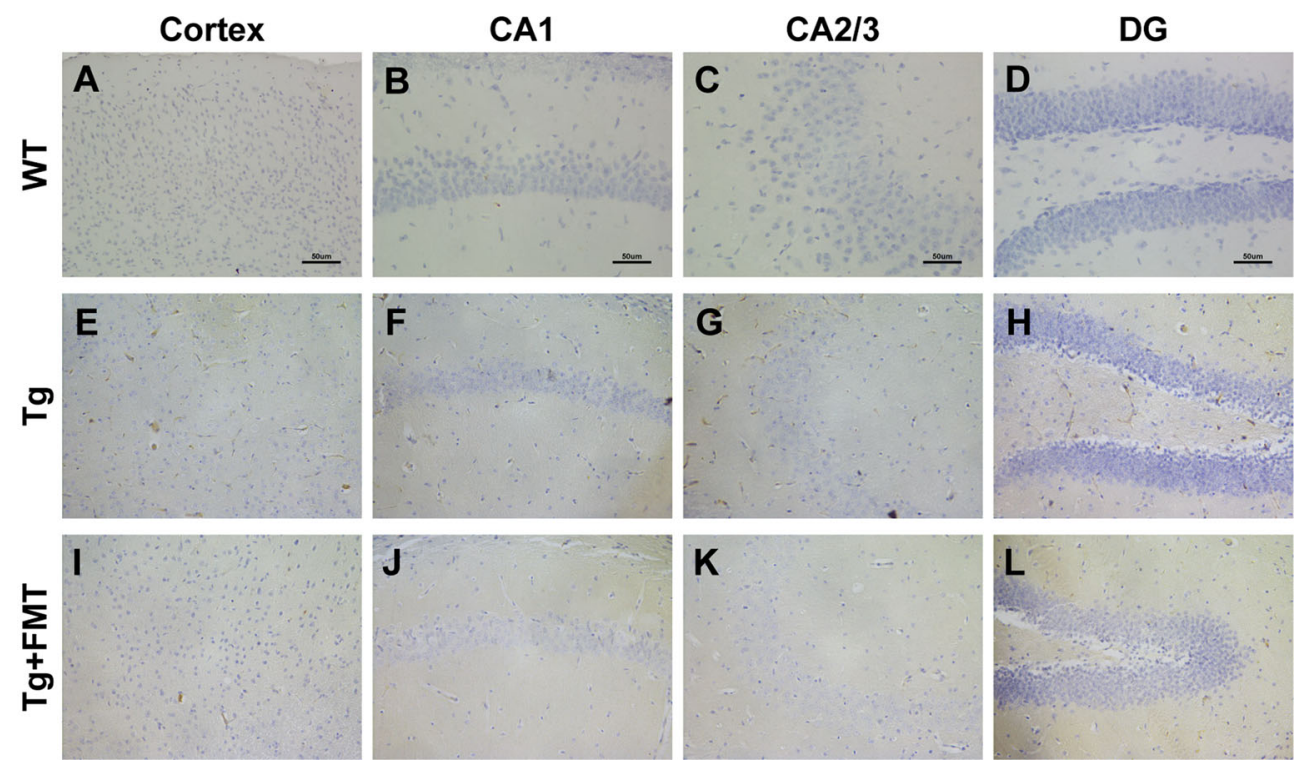

Fig. 3 Effects of FMT on levels of COX-2 and CD11b in Tg mice. a Immunohistochemical analysis of COX-2 in the cortex and hippocampal regions (CA1, CA3, and DG); Magnification $\times 400$. Scale bar $=50 \mu \mathrm{m}$. b Western blot analysis of COX-2 expression; c quantitative analysis of expression of COX-2, the reference value of the COX-2/GAPDH was the ratio of the WT group. Error bars indicate SEM; $n=4-6$ for each group. ${ }^{*} P<0.05$ vs. WT, ${ }^{\#} P<0.05$ vs. Tg. $\mathbf{b}$ Immunohistochemical analysis of CD1 1 b in the cortex and hippocampal regions (CA1, CA3, and DG); Magnification $\times 400$. Scale $\mathrm{bar}=50 \mu \mathrm{m}$ 


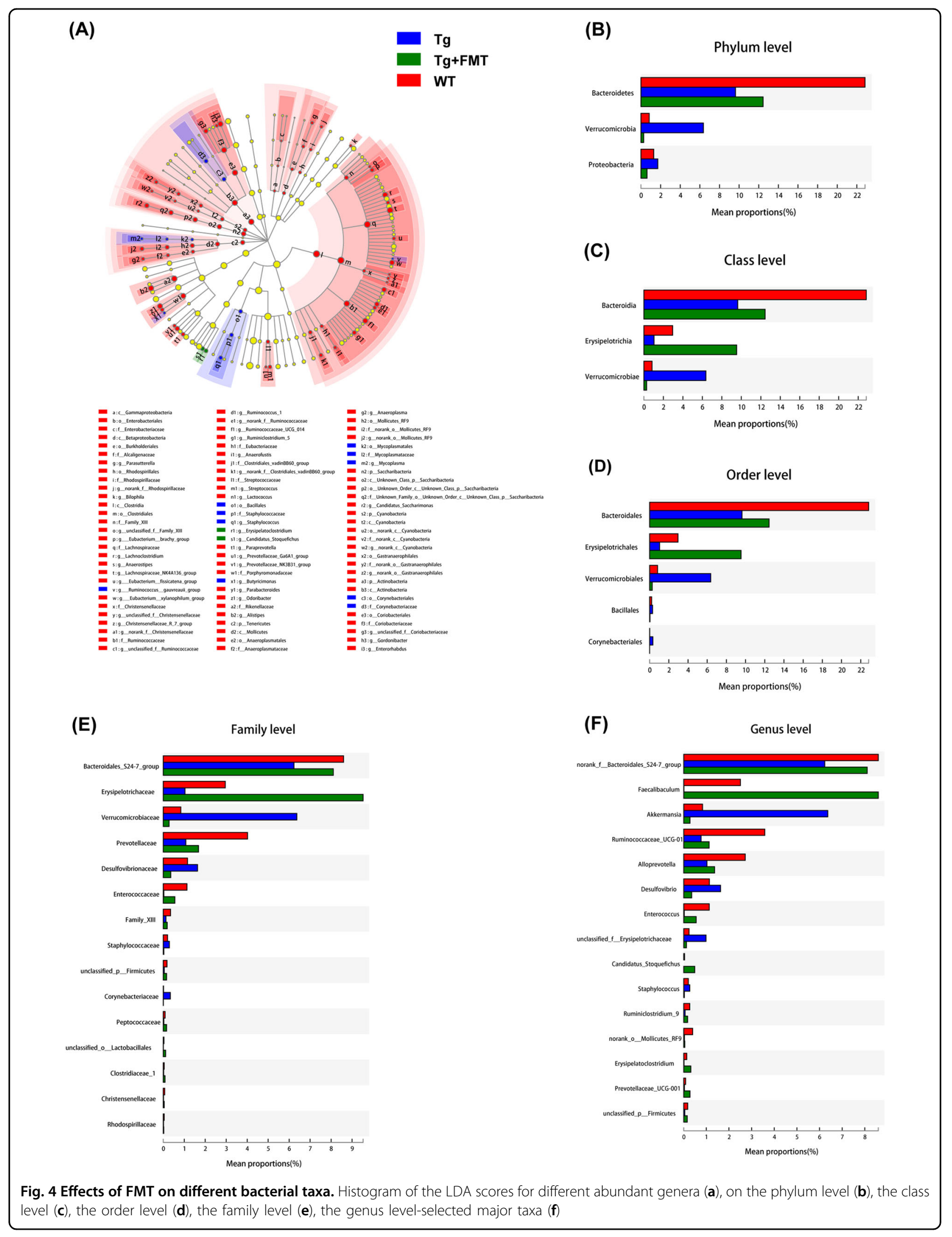




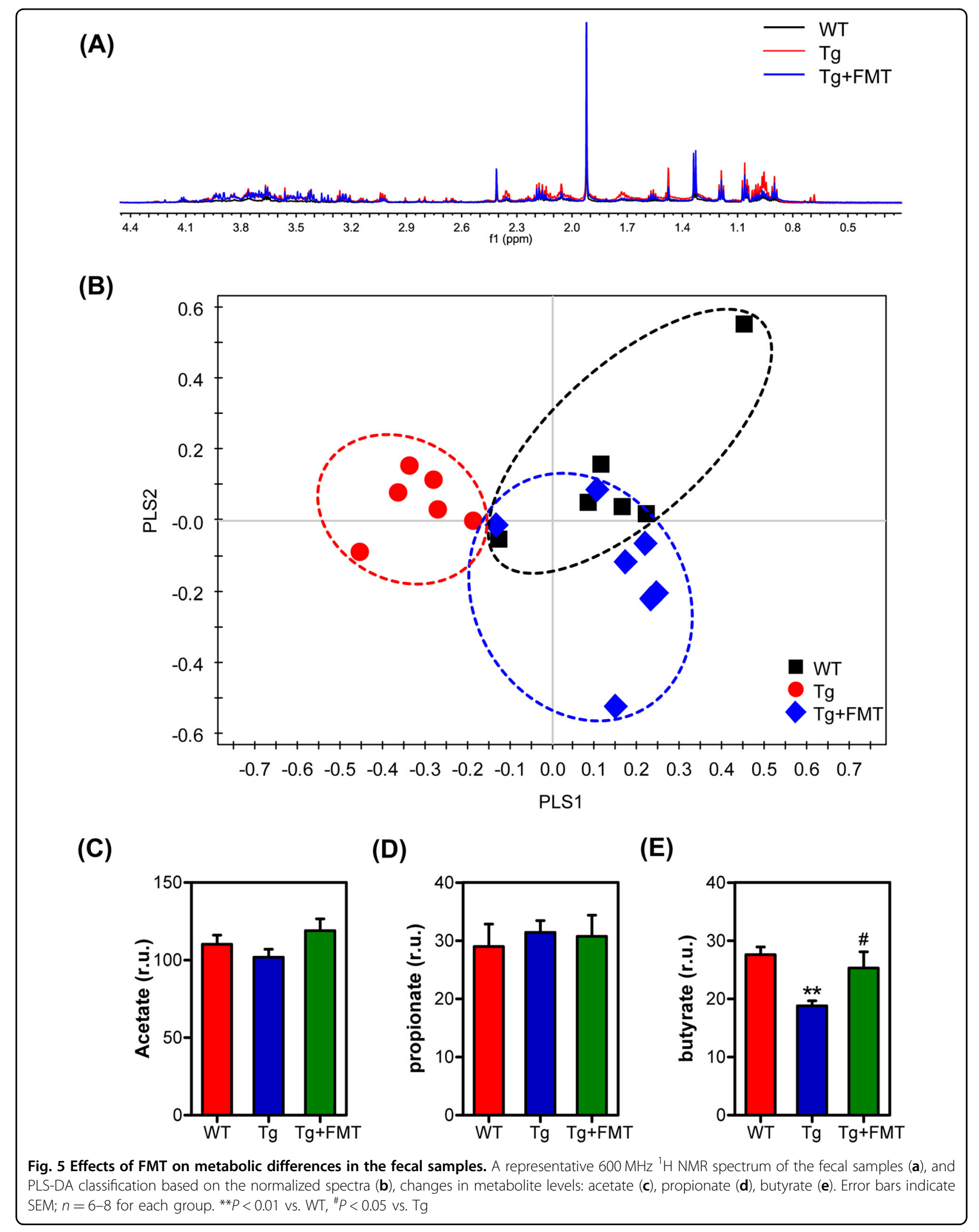


FMT treatment significantly reversed the decrease of butyrate in the Tg mice, suggesting the mechanism of anti-AD may be related to the increase of SCFAs butyrate. A further study to investigate this potential mechanism is necessary.

In summary, FMT prevented AD-like pathology in a mouse model, and these protective effects were related to reversing changes of gut microbiota and SCFAs, suggesting that FMT therapy might be a potential therapeutic strategy for $\mathrm{AD}$.

\section{Acknowledgements}

This work was supported by National Natural Science Foundation of China (No. 81871094).

\section{Author details}

'Department of Neurology, The Second Affiliated Hospital and Yuying Children's Hospital of Wenzhou Medical University, Wenzhou, Zhejiang 325027, China. ${ }^{2}$ Department of General Surgery, The Second Affiliated Hospital and Yuying Children's Hospital of Wenzhou Medical University, Wenzhou, Zhejiang 325027, China. ${ }^{3}$ Department of Pathophysiology, School of Basic Medicine Science, Wenzhou Medical University, Wenzhou, Zhejiang 325035, China. ${ }^{4}$ Department of Preventive Medicine, School of Public Health and Management, Wenzhou Medical University, Wenzhou, Zhejiang 325035, China

\section{Conflict of interest}

The authors declare that they have no conflict of interest.

\section{Publisher's note}

Springer Nature remains neutral with regard to jurisdictional claims in published maps and institutional affiliations.

Received: 13 February 2019 Revised: 22 April 2019 Accepted: 31 May 2019 Published online: 05 August 2019

\section{References}

1. Jin, W. et al. Peritoneal dialysis reduces amyloid-beta plasma levels in humans and attenuates Alzheimer-associated phenotypes in an APP/PS1 mouse model. Acta Neuropathol. 134, 207-220 (2017).

2. Zhu, C., Xu, B., Sun, X., Zhu, Q. \& Sui, Y. Targeting CCR3 to reduce amyloid- $\beta$ production, Tau hyperphosphorylation, and synaptic loss in a mouse model of Alzheimer's disease. Mol. Neurobiol. 54, 7964-7978 (2017).

3. Jiang, C., Li, G., Huang, P., Liu, Z. \& Zhao, B. The gut microbiota and Alzheimer's disease. J. Alzheimers Dis. 58, 1-15 (2017).

4. Zhuang, Z. Q. et al. Gut microbiota is altered in patients with Alzheimer's disease. J. Alzheimers Dis. 63, 1337-1346 (2018).

5. Shen, L., Liu, L. \& Ji, H. F. Alzheimer's disease histological and behavioral manifestations in transgenic mice correlate with specific gut microbiome state. J. Alzheimers Dis. 56, 385-390 (2017)

6. Pistollato, F. et al. Role of gut microbiota and nutrients in amyloid formation and pathogenesis of Alzheimer disease. Nutr. Rev. 74, 624-634 (2016).

7. Berezov, T. T., Kudinova, N. V. \& Kudinov, A. P. The role of Alzheimer amyloid plaques in the mechanisms of neuron synaptic plasticity disturbance. Vestn. Ross. Akad. Med. Nauk. 60, 3-7 (2005).

8. Akbari, E. et al. Effect of probiotic supplementation on cognitive function and metabolic status in Alzheimer's disease: a randomized, double-blind and controlled trial. Front. Aging Neurosci. 2016; 8, 256 (2016).

9. Bonfili, L. et al. SLAB51 probiotic formulation activates SIRT1 pathway promoting antioxidant and neuroprotective effects in an AD mouse model. Mol. Neurobiol. 55, 7987-8000 (2018).

10. Sun, J. et al. Clostridium butyricum attenuates chronic unpredictable mild stress-induced depressive-like behavior in mice via the gut-brain axis. J. Agric. Food Chem. 66, 8415-8421 (2018).
11. Liu, J. et al. Neuroprotective effects of Clostridium butyricum against vascular dementia in mice via metabolic butyrate. BioMed. Res. Int. 2015, 412946 (2015).

12. Sun, J. et al. Clostridium butyricum attenuates cerebral ischemia/reperfusion injury in diabetic mice via modulation of gut microbiota. Brain Res. 1642 180-188 (2016).

13. Li, H. et al. Clostridium butyricum exerts a neuroprotective effect in a mouse model of traumatic brain injury via the gut-brain axis. Neurogastroenterol. Motil. 30, e13260 (2018)

14. Han, A., Sung, Y. B., Chung, S. Y. \& Kwon, M. S. Possible additional antidepressant-like mechanism of sodium butyrate: targeting the hippocampus. Neuropharmacology 81, 292-302 (2014).

15. Schroeder, F. A., Lin, C. L., Crusio, W. E. \& Akbarian, S. Antidepressant-like effects of the histone deacetylase inhibitor, sodium butyrate, in the mouse. Biol. Psychiatry 62, 55-64 (2007)

16. Liu, J. et al. Sodium butyrate exerts protective effect against Parkinson's disease in mice via stimulation of glucagon like peptide-1. J. Neurol. Sci. 381, 176-181 (2017).

17. Sun, J. et al. Antidepressant-like effects of sodium butyrate and its possible mechanisms of action in mice exposed to chronic unpredictable mild stress. Neurosci. Lett. 618, 159-166 (2016).

18. Li, H. et al. Sodium butyrate exerts neuroprotective effects by restoring the blood-brain barrier in traumatic brain injury mice. Brain Res. 1642, 70-78 (2016).

19. Macfabe, D. F. Short-chain fatty acid fermentation products of the gut microbiome: implications in autism spectrum disorders. Microb. Ecol. Health Dis. 23, 19260 (2012).

20. Macfarlane, S. \& Macfarlane, G. T. Regulation of short-chain fatty acid production. Proc. Nutr. Soc. 62, 67-72 (2003).

21. Kang, D. et al. Microbiota Transfer Therapy alters gut ecosystem and improves gastrointestinal and autism symptoms: an open-label study. Microbiome 5, 10 (2017).

22. $\mathrm{Xu}, \mathrm{Z}$. et al. Fecal microbiota transplantation from healthy donors reduced alcohol-induced anxiety and depression in an animal model of chronic alcohol exposure. Chin. J. Physiol. 61, 360-371 (2018)

23. Yang, $\mathbf{C}$. et al. Key role of gut microbiota in anhedonia-like phenotype in rodents with neuropathic pain. Transl. Psychiatry 9, 57 (2019).

24. Sun, M. et al. Neuroprotective effects of fecal microbiota transplantation on MPTP-induced Parkinson's disease mice: gut microbiota, glial reaction and TLR4/TNF-a signaling pathway. Brain, Behav., Immun. 70, 48-60 (2018).

25. Wei, $Y$. et al. Fecal microbiota transplantation ameliorates experimentally induced colitis in mice by upregulating AhR. Front. Microbiol. 9, 1921 (2018).

26. Suez, J. et al. Artificial sweeteners induce glucose intolerance by altering the gut microbiota. Nature 514, 181-186 (2014)

27. Wu, Y. Y, Hsu, C. M, Chen, P. H. Fung, C. P. \& Chen, L. W. Toll-like receptor stimulation induces nondefensin protein expression and reverses antibioticinduced gut defense impairment. Infect. Immun. 82, 1994-2005 (2014).

28. Zhou, D. et al. Total fecal microbiota transplantation alleviates high-fat dietinduced steatohepatitis in mice via beneficial regulation of gut microbiota. Sci. Rep. 7, 1529 (2017).

29. Zheng, $\mathrm{H}$. et al. NMR-based metabolomics reveal a recovery from metabolic changes in the striatum of $6-\mathrm{OHDA}$-induced rats treated with basic fibroblast growth factor. Mol. Neurobiol. 53, 6690-6697 (2016).

30. Kim, T. K. et al. Analysis of differential plaque depositions in the brains of Tg2576 and Tg-APPswe/PS1dE9 transgenic mouse models of Alzheimer disease. Exp. Mol. Med. 44, 492-502 (2012).

31. Xin, Y. et al. Effects of oligosaccharides from Morinda officinalis on gut microbiota and metabolome of APP/PS1 transgenic mice. Front Neurol. 9, 412 (2018).

32. Sun, J. et al. Liraglutide improves water Maze learning and memory performance while reduces hyperphosphorylation of tau and neurofilaments in APP/PS1/Tau triple transgenic mice. Neurochem. Res. 42, 2326-2335 (2017).

33. Mancuso, C. \& Santangelo, R. Alzheimer's disease and gut microbiota modifications: the long way between preclinical studies and clinical evidence. Pharmacol. Res. 129, 329-336 (2018).

34. Nimgampalle, M. \& Kuna, Y. Anti-Alzheimer properties of probiotic, Lactobacillus plantarum MTCC 1325 in Alzheimer's disease induced albino rats. J. Clin. Diagn. Res. 11, KC01-KC05 (2017).

35. Wang, D. et al. beta2 adrenergic receptor, protein kinase A (PKA) and c-Jun Nterminal kinase (JNK) signaling pathways mediate tau pathology in Alzheimer disease models. J. Biol. Chem. 288, 10298-10307 (2013). 
36. Yao, Z., Yang, W., Gao, Z. \& Jia, P. Nicotinamide mononucleotide inhibits JNK activation to reverse Alzheimer disease. Neurosci. Lett. 647, 133-140 (2017).

37. Ramin, M., Azizi, P., Motamedi, F., Haghparast, A. \& Khodagholi, F. Inhibition of JNK phosphorylation reverses memory deficit induced by beta-amyloid (1-42) associated with decrease of apoptotic factors. Behav. Brain Res. 217, 424-431 (2011).

38. Cisse, M. et al. The transcription factor XBP1s restores hippocampal synaptic plasticity and memory by control of the Kalirin-7 pathway in Alzheimer model. Mol. Psychiatry 22, 1562-1575 (2017).

39. Koss, D. J., Drever, B. D., Stoppelkamp, S., Riedel, G. \& Platt, B. Age-dependent changes in hippocampal synaptic transmission and plasticity in the PLB1 Triple Alzheimer mouse. Cell Mol. Life Sci. 70, 2585-2601 (2013).

40. Sultana, R., Banks, W. A. \& Butterfield, D. A. Decreased levels of PSD95 and two associated proteins and increased levels of $\mathrm{BCl}_{2}$ and caspase 3 in hippocampus from subjects with amnestic mild cognitive impairment: insights into their potential roles for loss of synapses and memory, accumulation of Abet. J. Neurosci. Res. 88, 469-477 (2010).

41. Benarroch, E. E. Glutamatergic synaptic plasticity and dysfunction in Alzheimer disease: emerging mechanisms. Neurology 91, 125-132 (2018).

42. Skaper, S. D., Facci, L., Zusso, M. \& Giusti, P. Synaptic plasticity, dementia and Alzheimer disease. CNS Neurol. Disord. Drug Targets 16, 220-233 (2017).

43. Shankar, G. M. et al. Amyloid-beta protein dimers isolated directly from Alzheimer's brains impair synaptic plasticity and memory. Nat. Med. 14, 837-842 (2008).

44. Piserchio, A., Spaller, M. \& Mierke, D. F. Targeting the PDZ domains of molecular scaffolds of transmembrane ion channels. AAPS J. 8, E396-E401 (2006).

45. Marsh, J., Bagol, S. H., Williams, R. S. B., Dickson, G. \& Alifragis, P. Synapsin I phosphorylation is dysregulated by beta-amyloid oligomers and restored by valproic acid. Neurobiol. Dis. 106, 63-75 (2017).

46. Larson, M. E. et al. Selective lowering of synapsins induced by oligomeric alpha-synuclein exacerbates memory deficits. Proc. Natl Acad. Sci. USA 114, E4648-E4657 (2017).

47. Woo, J. Y. et al. Lactobacillus pentosus var. plantarum C29 ameliorates memory impairment and inflammaging in a D-galactose-induced accelerated aging mouse model. Anaerobe 27, 22-26 (2014).

48. Aïd, S. \& Bosetti, F. Targeting cyclooxygenases-1 and -2 in neuroinflammation: therapeutic implications. Biochimie 93, 46-51 (2011).

49. Petrov, V. et al. Analysis of gut microbiota in patients with Parkinson's disease. Bull. Exp. Biol. Med. 162, 734-737 (2017).

50. Scheperjans, F. et al. Gut microbiota are related to Parkinson's disease and clinical phenotype. Mov. Disord. 30, 350-358 (2015).

51. Zhang, $L$. et al. Altered gut microbiota in a mouse model of Alzheimer's disease. J. Alzheimers Dis. 60, 1241-1257 (2017).

52. Cattaneo, A. et al. Association of brain amyloidosis with pro-inflammatory gut bacterial taxa and peripheral inflammation markers in cognitively impaired elderly. Neurobiol. Aging 49, 60-68 (2017).

53. Minter, M. R. et al. Antibiotic-induced perturbations in microbial diversity during post-natal development alters amyloid pathology in an aged APPSWE/ PS1 $\triangle$ E9 murine model of Alzheimer's disease. Sci. Rep. 7, 10411 (2017).
54. OR, J. A. et al. Modulation of intestinal microbiota by the probiotic VSL\#3 resets brain gene expression and ameliorates the age-related deficit in LTP. PloS ONE 9, e106503 (2014).

55. Biagi, E. et al. Through ageing, and beyond: gut microbiota and inflammatory status in seniors and centenarians. PLoS ONE 5, e10667 (2010).

56. Bauerl, C., Collado, M. C., Diaz Cuevas, A., Vina, J. \& Perez Martinez, G. Shifts in gut microbiota composition in an APP/PSS1 transgenic mouse model of Alzheimer's disease during lifespan. Lett. Appl. Microbiol. 66, 464-471 (2018).

57. Shin, N. R., Whon, T. W. \& Bae, J. W. Proteobacteria: microbial signature of dysbiosis in gut microbiota. Trends Biotechnol. 33, 496-503 (2015).

58. Chen, C. H., Lin, C. L. \& Kao, C. H. Irritable bowel syndrome Is associated with an increased risk of dementia: a nationwide population-based study. PLOS ONE 11, e0144589 (2016).

59. Wang, J. et al. The effects of LW-AFC on intestinal microbiome in senescenceaccelerated mouse prone 8 strain, a mouse model of Alzheimer's disease. J. Alzheimers Dis. 53, 907-919 (2016).

60. Malkki, H. Parkinson disease: Could gut microbiota influence severity of Parkinson disease? Nat. Rev. Neurol. 13, 66-67 (2017).

61. Debelius, J. et al. Parkinson's disease and Parkinson's disease medications have distinct signatures of the gut microbiome. Mov. Disord. 32, 739-749 (2017).

62. Pandey, U. et al. The nasal and gut microbiome in Parkinson's disease and idiopathic rapid eye movement sleep behavior disorder. Mov. Disord. 33, 88-98 (2018).

63. Harach, T. et al. Erratum: Reduction of Abeta amyloid pathology in APPPS1 transgenic mice in the absence of gut microbiota. Sci. Rep. 7, (46856 (2017).

64. Vogt, N. M. et al. Gut microbiome alterations in Alzheimer's disease. Sci. Rep. 7 13537 (2017).

65. Brandscheid, C. et al. Altered gut microbiome composition and tryptic activity of the 5xFAD Alzheimer's mouse model. J. Alzheimers Dis. 56, 775-788 (2017).

66. He, Y. et al. Gut microbiome and magnetic resonance spectroscopy study of subjects at ultra-high risk for psychosis may support the membrane hypothesis. Eur. Psychiat. 53, 37-45 (2018).

67. Varela, R. B. et al. Sodium butyrate and mood stabilizers block ouabaininduced hyperlocomotion and increase BDNF, NGF and GDNF levels in brain of Wistar rats. J. Psychiatr. Res. 61, 114-121 (2015).

68. Erny, D. et al. Host microbiota constantly control maturation and function of microglia in the CNS. Nat. Neurosci. 18, 965-977 (2015).

69. MacFabe, D. F. et al. Neurobiological effects of intraventricular propionic acid in rats: possible role of short chain fatty acids on the pathogenesis and characteristics of autism spectrum disorders. Behav. Brain Res. 176, 149-169 (2007).

70. Ho, L. et al. Protective roles of intestinal microbiota derived short chain fatty acids in Alzheimer's disease-type beta-amyloid neuropathological mechanisms. Expert Rev. Neurother. 18, 83-90 (2018).

71. Griseri, P. et al. Rescue of human RET gene expression by sodium butyrate: a novel powerful tool for molecular studies in Hirschsprung disease. Gut 52, 1154-1158 (2003)

72. Sun, J. et al. Neuroprotective effect of sodium butyrate against cerebral ischemia/reperfusion injury in mice. BioMed. Res. Int. 2015, 395895 (2015). 